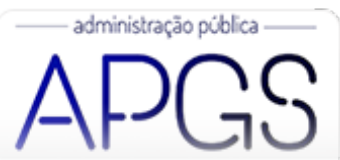

Administração Pública e Gestão Social ISSN: 2175-5787

apgs@ufv.br

Universidade Federal de Viçosa

Brasil

\title{
Retos de futuro en la gestión de los recursos humanos públicos en España
}

\author{
Pineda Nebot, Carmen \\ Retos de futuro en la gestión de los recursos humanos públicos en España \\ Administração Pública e Gestão Social, vol. 11, núm. 4, 2019 \\ Universidade Federal de Viçosa, Brasil \\ Disponible en: http://www.redalyc.org/articulo.oa?id=351560525002
}

Esta obra está bajo una Licencia Creative Commons Atribución-NoComercial-SinDerivar 3.0 Internacional. 


\section{Retos de futuro en la gestión de los recursos humanos públicos en España}

Future challenges in the management of public human resources in Spain

Desafios futuros na gestão de recursos humanos públicos na Espanha

Carmen Pineda Nebot

Universidad Autónoma de Madrid, España

Redalyc: http://www.redalyc.org/articulo.oa?

camenpinedanebot@hotmail.com $\mathrm{id}=351560525002$

Recepción: 26 Enero 2019

Aprobación: 13 Agosto 2019

Publicación: 01 Octubre 2019

\section{RESUMEN:}

El objetivo de este trabajo es conocer los retos de futuro a los que se enfrentan los países en la gestión de los recursos humanos públicos, a partir de la experiencia española. Las políticas públicas actuales cada vez más complejas, interconectadas, volátiles y fragmentadas junto con las nuevas herramientas tecnológicas hacen cada vez más necesario un nuevo perfil de empleados públicos, empleados que deben ser innovadores, con orientación estratégica, flexibles, creativos y motivados. Para ello es preciso, y así lo están planteando muchos expertos, modificar tanto los sistemas de selección de nuevos talentos como de formación de los existentes. Es importante, por tanto, que se utilice una gestión estratégica de los recursos humanos, gestión que debe tener en cuenta las especiales características de lo público.

Palabras Clave: gestión de personas, selección, formación.

\section{Abstract:}

This study's aim is to know the future challenges facing countries in the management of public human resources, based on the Spanish experience. The current public policies increasingly complex, interconnected, volatile and fragmented together with the new technological tools makes a new profile of public employees increasingly necessary, employees who must be innovative, with strategic orientation, flexible, creative and motivated. For this, it is necessary, and that is what many experts are asking, to modify both the systems for the selection of new talents and the training of existing ones. It is important, therefore, that a strategic management of human resources is used, that must take into account the special characteristics of the public.

KEYWORDS: people management, selection, training.

\section{Resumo:}

O objetivo deste trabalho é conhecer os desafios futuros que os países enfrentam na gestão de recursos humanos públicos, com base na experiência espanhola. As atuais políticas públicas cada vez mais complexas, interligadas, voláteis e fragmentadas aliadas às novas ferramentas tecnológicas tornam cada vez mais necessário um novo perfil de servidores públicos, funcionários que devem ser inovadores, com orientação estratégica, flexíveis, criativos e motivados. Para isso, é necessário modificar tanto os sistemas para a seleção de novos talentos quanto para o treinamento dos existentes, e é isso que muitos especialistas estão pedindo. É importante, portanto, que seja utilizada uma gestão estratégica dos recursos humanos, gestão que deve levar em conta as características especiais do público.

PalaVRaS-ChaVE: gestão de pessoas, seleção, formação.

\section{INTRODUCCIÓN}

Las políticas públicas actuales son cada vez más complejas, interconectadas, volátiles y fragmentadas, mientras que las herramientas tecnológicas y las posibilidades del trabajo con múltiples actores evolucionan a una velocidad sin precedentes (Organización para la Cooperación y el Desarrollo Económico - OCDE, 2017). En este nuevo contexto se necesita un nuevo perfil de empleados públicos ${ }^{[\mathrm{i}]}$, que deben ser innovadores, con orientación estratégica, flexibles, creativos y motivados. 
La eficiencia de los gobiernos, su capacidad para proveer eficazmente servicios públicos y la agilidad necesaria para implementar las políticas, depende mucho de las capacidades de sus empleados, contribuyendo de una manera significativa a ello una buena gestión de los recursos humanos. La gestión pública de recursos humanos tiene como fin proveer a la organización administrativa de las capacidades necesarias, de las personas con los conocimientos, las destrezas, las habilidades y las actitudes necesarias en el lugar y momento demandados (Cuenca, 2018).

El problema no es solo que antes y durante las reciente crisis económicas la estrategia en materia de recursos humanos en la administración pública haya brillado por su ausencia; lo que resulta preocupante es que después de la crisis no se vaya a iniciar un mínimo proceso de reflexión crítica sobre qué tipo de Administración Pública y de funcionarios públicos se van a necesitar en una sociedad que está experimentando una rápida transformación y que va a requerir de sus administraciones la prestación de unos servicios muy distintos de los actuales, lo que lógicamente supone la necesidad de un capital humano también muy distinto (Nuez, 2018).

$\mathrm{Si}$ a los cambios tecnológicos y organizativos se une el relevo generacional que se plantea, en la administración española, a causa del envejecimiento de sus plantillas, el problema se agudiza. Nos encontramos unas plantillas públicas notablemente envejecidas y que van a generar un fenómeno de jubilaciones masivas y por tanto una notable descapitalización en términos de conocimiento organizativo (Gorriti, 2016).

España se enfrentará en los próximos años a un relevo generacional muy importante (Jiménez Asensio y Gorriti, 2016; Gorriti, 2015; Jiménez Asensio, 2018; Ramió y Salvador, 2018). Las muy numerosas cohortes de funcionarios que ingresaron en la Administración Pública durante los años 80 y 90 del siglo pasado momento en el cual se pusieron en marcha las diferentes Administraciones autonómicas con un modelo básicamente idéntico al estatal en lo que a la política de recursos humanos se refiere - se irán jubilando ${ }^{[i i]}$ a lo largo de las dos próximas décadas ${ }^{[i i i]}$. En los últimos 15 años la edad media de la plantilla en la Administración General del Estado ha pasado de 46 años en 2004 a 52 años en 2019, según datos oficiales. El 15\% del personal funcionario del estado ya tiene más de 60 años y solo el 10\% tiene menos de 40 años. (Error 57: La referencia debe estar ligada) (Error 58: El tipo de referencia es un elemento obligatorio) (Error 59: No existe una url relacionada)

Esta coyuntura ofrece la gran oportunidad de un cambio incremental no traumático en la administración pública en la medida en que permite un drástico cambio de paradigma sin tener que enfrentarse con las lógicas resistencias internas de unos funcionarios o empleados públicos cuyas competencias, formación o capacidades ya no son las adecuadas para atender a las necesidades públicas de la Administración del siglo XXI.

Debe comenzar, por tanto, un gran proceso de reflexión colectiva en clave estratégica sobre el modelo de empleo público en las sociedades, en el que se requiere "diagnosticar adecuadamente el problema en el marco de un plan estratégico y adoptar medidas estructurales e institucionales que transformen la función pública, la renueven e introduzcan elementos propios de innovación, flexibilidad, creatividad y adaptabilidad como ejes de su actuación, con la mirada puesta en los servicios públicos (misiones) que el sector público deberá prestar en los próximos veinticinco o treinta años (que también mutarán de forma importante)" (Gorriti y Jiménez Asensio, 2016).

Con el fin de aportar algo más sobre el tema en discusión se expone en el artículo, en primer lugar, el marco teórico de partida; seguidamente, presentamos la metodología utilizada; a continuación, se analizan tres elementos de la gestión de recursos humanos públicos: número y tipos de empleados, selección de empleados públicos y la formación de estos; y, por último, se formulan algunas conclusiones. 


\section{MARCO TEÓRICO}

El empleo público es considerado aquí en su sentido más amplio, como ocurre en los textos internacionales que tratan del tema, como los de la Organización Internacional del Trabajo (OIT), los de la Organización para la Cooperación y el Desarrollo Económico (OCDE) y también los del Banco Mundial. Lo que significa que el empleo público incluye en el caso de España no solo a los funcionarios sino también a los laborales y a los eventuales de todos los niveles de gobierno.

Pero no podemos olvidar que las organizaciones no actúan en el vacío, existe un contexto que conviene tener presente, analizar y tomar posición ante el mismo, una vez analizada su incidencia y el estado de la propia organización, para lo que se fijan una serie de prioridades de actuación. Es por tanto un proceso analítico, intelectual, que a partir de un diagnóstico de situación, concreta con los actores relevantes cuáles son los temas críticos cuya consecución permitirá estimar el éxito o fracaso de la acción de gobierno, de la efectiva creación de valor público (Cuenca, 2018). Se ordena la complejidad del entorno cuando se fija una estrategia ante el mismo (Morin, 2011, p. 114).

La planificación estratégica aplicada a la política de personal constituye la contextualización de una técnica, la gestión de recursos humanos a una realidad administrativa concreta, mediante el análisis de los factores del entorno, el contraste con la situación de sus efectivos y la delimitación de las líneas de acción a emprender. El primer nivel en la planificación sería fijar tanto el número de efectivos como su estructura. Planificar es así un camino tendente a adecuar la estructura social existente a la meta pretendida. Se deben establecer las líneas de evolución a medio y largo plazo de la fuerza laboral a partir de una reflexión que tome en consideración los factores condicionantes de orden político, económico, social y tecnológico - los conocidos factores PEST (Políticos, Económicos, Sociales y Tecnológicos) —, su proyección en el tiempo y su determinación mediante escenarios que estimen su impacto sobre los sistemas de organización del trabajo.

Cantidad y composición de la fuerza laboral, su progresión y movilidad, así como la innovación de los sistemas que permiten gestionarla eficazmente, conforman la política de empleo público a medio y largo plazo. Pero esta operación está condicionada a su vez por dos factores que la limitan: los ineludibles escenarios económico-financieros y el inventario de servicios públicos que provea la organización. Lo financieramente sostenible condiciona la gestión, por lo que son el antecedente inmediato de cualquier transformación o proceso de redimensionamiento. El gasto público es el factor que incide a su vez sobre el segundo elemento conformador de las políticas de personal: el inventario de servicios públicos. Si como se sostiene, la piedra angular de la política de personal es el dimensionamiento de efectivos, este a su vez es inseparable de los servicios y políticas públicas que la Administración decide legítimamente ofertar, así como del modelo organizativo que establezca (Cuenca,2018).

La plantilla el componente subjetivo, se ordena - o se ajusta- siempre con la vista puesta en las necesidades de los servicios, que son las que priman y enmarcan así la relación del empleado público y su contenido material. La gestión pública de personas no tiene lugar en el vacío. Existe un marco organizativo, integrado por los servicios públicos y por los elementos que intervienen en su demanda (el público objetivo, la coherencia de la oferta o la satisfacción con su producción por parte de los usuarios, entre otros aspectos). Fijado el inventario/catálogo/cartera de los servicios, programas y políticas a prestar es cuando procede el diseño de la estructura organizativa y sus bases de agrupación, así como decisiones estratégicas relacionadas con su provisión (por medios directos o externalizados) (Cuenca, 2018).

Pese a su poca presencia efectiva, la planificación de recursos humanos no es algo desconocido, al menos en términos teóricos, en la tradición administrativa española. En 2007 el Estatuto Básico del Empleado Público (EBEP), a través de su artículo 69, contempló un propósito ambicioso para la planificación estratégica que iba más allá de su antecedente, los "planes de empleo" instituidos en el periodo de 1993 hasta 1995 (Cuenca, 2018). Se trataba de un instrumento que debía servir para configurar - tal y como recogía la propia exposición de motivos del EBEP — "su propia política de personal" que cada Administración 
podía decidir en uso de su potestad de autoorganización. A pesar de ello la última reflexión al respecto, la procedente del Informe CORA (2013), recomienda nuevamente reforzar la vinculación entre las necesidades de personal y los objetivos estratégicos de la organización, en un reconocimiento de su falta de aplicación. En la Administración Española sigue sin implantarse de modo efectivo un enfoque sostenido de gestión a medio plazo, circunstancia a la que no es ajena la dificultad de arraigo de la propia gestión pública de recursos humanos como disciplina aplicada a un entorno institucional, el público, caracterizado por unos criterios de éxito distintos al del mercado, sociales y políticos además de económicos, y con unos actores singulares políticos y sindicales - y un marco jurídico que trata de asegurar valores específicos como el mérito (Cuenca, 2018, p. 55).

Para los gestores públicos supone una gran dificultad contar de manera explícita con lo que hemos denominado los factores conformadores de la planificación de recursos humanos: el marco económico presupuestario y el planteamiento en relación con la cartera de servicios, lo que viene a ser tanto como la ausencia de objetivos a medio plazo. Esto los sitúa en lo que Longo (2004, p. 97) ha denominado "el dilema de la estrategia”. La política de recursos humanos debe estar alineada con los objetivos estratégicos de la organización. Ausentes estos, un plan de ordenación no cuenta con los elementos mínimos para su concreción (Cuenca, 2018).

Por ello hay que crear una metodología para actuar a priori como planificación organizativa. Concretando una secuencia que se debe iniciar ya, independientemente del número de jubilaciones que se tengan o se prevean, es una necesidad obvia que exige tener planificado el empleo público antes de que ocurran. Debe saberse: a) qué puestos hay que diseñar; b) cuáles hay que rediseñar; y c) cuáles no reponer. Para llegar a estos productos hay dos vías que se entrecruzan solo al final: la vía estratégica (política con asesoramiento técnico organizativo), que es la parte puramente organizativa y tiene a las funciones como materia prima; las responsabilidades que se asumen y crean, matizan o no justifican la existencia de puestos. La segunda vía se refiere a las tareas: los procesos que satisfacen las responsabilidades asumidas en las funciones; la traslación que a la realidad de las ejecuciones hacen las personas (esencia de los RRHH) (Gorriti y Jiménez Asensio, 2018).

Pero hablar de estrategia no solo es redactar documentos formales sobre planificación sino que es sobre todo referirse a una perspectiva compartida entre los miembros de una organización. No siendo procesos sencillos para llevar a cabo en determinados contextos administrativos. Por ello los planteamientos de planificación de recursos humanos pos burocráticos deben prestar tanta atención a las variables de proceso relacionadas con el impulso político-directivo (el liderazgo) como a los de contenido (Cuenca, 2018). Respecto a este último aspecto, una estrategia viable debe, además de fundarse en un diagnóstico sólido basado en datos, alcanzar una visión global de los temas de personal, concretando el talento requerido a medio plazo y con la movilidad interna y la flexibilidad como eje ordenador. El énfasis radicaría así en disponer de una estrategia más concebida como brújula que como mapa (Cuenca, 2018).

Se trataría de que cada Administración contara con un documento sintético que, sin renunciar a una mayor concreción posterior, estableciera la estrategia a seguir en relación con su empleo público, mediante un discurso de valores públicos y una visión inspiradora a medio y largo plazo, una suerte, de directrices de política de personal en el que se fijarán las reglas de gestión y las líneas de orientación aplicables, los objetivos estratégicos y los concretos criterios políticos, que serviría no tanto para producir efectos jurídicos como para fijar la agenda política de temas a abordar sobre el servicio público.

También es especialmente importante ver que el mundo de las funciones y el de las tareas no se mezclan, y que las nuevas tecnologías sólo influyen sobre las tareas, no sobre las responsabilidades, aunque no se nos escapa que como todo proceso dialéctico en algún momento puede dar argumentos para una reflexión estratégica cualitativa. También es especialmente importante ver que, en realidad, sólo las funciones innecesarias son las que justifican la no reposición de vacantes, y que esta es una decisión estrictamente estratégica (política) tras una reflexión solo de técnica organizativa (Gorriti y Jiménez Asensio, 2018). 
Una herramienta importante de la gestión de recursos humanos, los procesos selectivos de acceso a las administraciones públicas, puede aportar muchos réditos, para abordar realidades actuales de interinidad y de cercanas jubilaciones masivas de funcionario, si se utiliza estratégicamente y como parte de un sistema integrado de organización y gestión de la diferencia. Esta concepción estratégica de la selección debe estar complementada por una visión científica de estos procesos donde la fiabilidad y la validez de las pruebas obtenidas por estudios propios, aseguren la factibilidad, justificación y adecuación de los candidatos/as a lo que van a hacer o aprender. También por una nueva concepción de los cuerpos de funcionarios y por la trascendente distinción entre persona y puesto. Como señala Gorriti (2018) "cuanto más se parezca el proceso selectivo al trabajo para el cual se hace la selección, mayor será la probabilidad de que dicho proceso selectivo sea válido y justo".

Ajustarse a las nuevas situaciones requiere adquirir otras capacidades que hasta ahora no eran necesarias. Desaprender mucho de lo aprendido y amoldarnos a la nueva realidad. Para ello, es fundamental la conectividad, no sólo entendida como la conexión entre las personas o entre las máquinas sino también como la capacidad de interacción entre ambas (Tamames, 2018).

Capacidades que se pueden adquirir, en el caso de no poseerlas, mediante la formación. Materia a la que no se ha prestado mucha atención en España, a pesar de la importancia intrínseca que tiene sobre la gestión de recursos humanos en las Administraciones Públicas ${ }^{[i v]}$. Habiendo dos grandes formas o modos de entender la formación de los empleados públicos, que no son exclusivos ni excluyentes: la primera puede referirse a la formación como acreditación de competencias, mientras que la segunda la podemos encuadrar en la línea de la formación y el análisis de puestos de trabajo (Jiménez Asensio, 2010).

Se utilice una u otra forma, lo cierto es que si realmente se quiere caminar hacia la articulación de un nuevo sistema de gestión de personas en las Administraciones Públicas españolas es un presupuesto imprescindible configurar la formación como una obligación o responsabilidad de los empleados públicos derivada consustancialmente de su relación con la Administración. Sin perder de vista que la formación de empleados públicos tiene una finalidad institucional como es coadyuvar a la efectividad del principio de buena administración y, por consiguiente, a mejorar la prestación de servicios a los ciudadanos (Jiménez Asensio, 2010). Por consiguiente, se debe reforzar la dimensión objetiva de la formación en plena coherencia con la institución sobre la que despliega sus efectos.

En cuanto a las dinámicas vinculadas a la adquisición de las nuevas competencias que necesitan las administraciones del futuro, se plantea como esencial la combinación de acciones formativas con la generación de actividades que faciliten tanto su configuración concreta como su desarrollo efectivo adaptado a la realidad organizativa. Los nuevos escenarios formativos que se planteen deben estar asociados especialmente a la formación on line, a la formación colaborativa, al autoaprendizaje y al aprendizaje informal (Cofer, 2000, Lohman, 2005 y 2009 ), además de relacionarse con una formación más personalizada y adecuada a las necesidades de los empleados públicos.

Respecto a los nuevos tipos de formadores, en este aspecto parece sugerente la figura del formador en red que deberá disponer de nuevas competencias y hacer uso de recursos innovadores. El nuevo perfil competencial del profesor debe estar asociado a un dinamizador, que incorpora diversos roles relacionados, tanto con habilidades blandas asociadas con la inteligencia emocional, como con habilidades propiamente docentes y también tecnológicas. Los roles que ha de desempeñar son como conector de personas, impulsor de escenarios de colaboración, de participación y co-creación, preservador de contenidos y facilitador de aprendizajes. Para el desarrollo de estos roles ha de dominar tanto las herramientas presenciales como digitales. 


\section{METODOLOGÍA}

El objetivo de la investigación es conocer, mediante el análisis de varias dimensiones, la política de recursos humanos públicos en España y los retos a los que esta se debe enfrentar. Para la consecución de este objetivo se ha partido de un análisis bibliográfico (Selltiz et al., 1980; Hernandez Sampieri et al., 2006) con el fin de conocer anteriores investigaciones sobre el tema y poder establecer así el marco teórico de referencia para encuadrar el trabajo. A su vez se ha realizado la revisión documental del marco legislativo para comprobar cómo se responde desde el marco legal a la política de recursos humanos en España. Además se han utilizado los datos sobre los efectivos al servicio de las distintas Administraciones Públicas facilitados por el Registro Central de Personal del Ministerio de Política Territorial y Función Pública. Semestralmente este Registro elabora, desde 1990, un Boletín Estadístico (BEPSAP) del personal al servicio de las Administraciones Públicas.

En cuanto al contexto, señalar que España es un país miembro de la Unión Europea, constituido en Estado social y democrático de derecho y cuya forma de gobierno es la monarquía parlamentaria. El producto interior bruto coloca a la economía española en la decimotercera posición mundial, siendo una potencia turística, pues es el segundo país más visitado del mundo, con 82 millones de turistas en 2017, y el segundo país del mundo en ingresos económicos provenientes del turismo. Su índice de desarrollo humano es muy alto (0.891), según el informe de 2017 del Programa de la ONU para el Desarrollo. España es en la actualidad lo que se denomina un "Estado de las autonomías" o "Estado autonómico", organizado territorialmente en diecisiete comunidades autónomas y dos ciudades autónomas, cada una de ellas con diferentes niveles de autogobierno. Estando considerado, hoy en día, como uno de los países europeos más descentralizados, ya que todos sus diferentes territorios administran de forma autonómica sus sistemas sanitarios y educativos, así como otro aspectos del presupuesto público; algunos de ellos, como el País Vasco y Navarra, además administran su financiación pública sin casi contar (a excepción del cupo) con la supervisión del gobierno central español. En el caso de Cataluña, Canarias, Navarra y el País Vasco, están además equipados con sus propios cuerpos policiales, totalmente operativos y completamente autónomos que reemplazan las funciones de la Policía Nacional en estos territorios. En cuanto al modelo de administración imperante se puede considera que conviven, en mayor o menor medida, elementos de tres modelos: el burocrático el modelo gerencial (con especial incidencia de la denominada Nueva Gestión Pública) y el modelo de gobernanza.

La gestión de recursos humanos es un conjunto de actividades planteadas sobre los empleados que desarrollan su actividad profesional en las Administraciones Públicas, que se puede visualizar desde una doble perspectiva, la de la gestión de personal y la de la gestión de personas. La gestión de personal, que es la que hemos analizado, hace referencia a la perspectiva macro o agregada, al conjunto de ámbitos de actuación o subsistemas (selección, formación, carrera administrativa, retribución, etc.) que constituyen el núcleo clásico de las aproximaciones los recursos humanos, asociado a un importante componente técnico. Por el contrario, la visión de la gestión de personas es una visión más micro, más cercana las actividades que los directivos de línea desarrollan para con los empleados que tiene adscritos y a la motivación e implicación de éstos. Como señala Grandinetti (2003, p. 196) la gestión de recursos humanos incluye "una serie de técnicas, procesos y actividades interrelacionadas, consistentes entre sí, las cuales, en su conjunto de algún modo explicitan a los empleados lo que se espera de ellos en función de los objetivos planteados para la organización”.

El análisis lo hemos realizado a partir del análisis de tres dimensiones importantes de la gestión de personal: el número y tipo de empleados, la selección de empleados públicos y la formación de estos. Entendiendo que cuando hablamos de personal nos estamos refiriendo al conjunto de efectivos que prestan sus servicios en: a) la Administración Pública Estatal, agrupando bajo esta rúbrica a: la Administración General del Estado, las Fuerzas y Cuerpos de Seguridad del Estado, las Fuerzas Armadas, la Administración de Justicia, Entidades Públicas Empresariales y Organismos Públicos con régimen específico; b) las Administraciones de las Comunidades Autónomas; c) la Administración Local, que agrupa a: Ayuntamientos y otras entidades 
locales y sus Organismos Autónomos y Diputaciones, Cabildos y Consejos Insulares y sus Organismos Autónomos y d) las Universidades.

Las tipologías de personal que hemos considerado son: a) Personal funcionario de carrera: es el que, en virtud de nombramiento legal, está vinculado a una Administración Pública por una relación estatutaria regulada por el Derecho Administrativo para el desempeño de servicios profesionales retribuidos de carácter permanente; b) Personal estatutario de los Servicios de Salud: es el que, en virtud de un nombramiento legal, presta servicios en los centros e instituciones sanitarias que conforman el Sistema Nacional de Salud y está sujeto a una relación funcionarial especial; c) Personal laboral: es el que en virtud de contrato de trabajo formalizado por escrito en cualquiera de las modalidades de contratación de personal previstas en la legislación laboral, presta servicios retribuidos por las Administraciones Públicas; d) Otro personal, que comprende: al eventual, al funcionario interino y al funcionario de plazas no escalafonadas (este personal está en extinción según la Ley 30/1984, de 2 de agosto); e) Funcionarios en formación o prácticas: son los aspirantes que, tras superar las pruebas selectivas, deben superar un periodo de prácticas o curso selectivo antes de ser nombrados funcionarios de carrera.

\section{RESULTADOS}

a) Número de empleados públicos

La proporción de personas que trabajan en el sector público ofrece una indicación de la manera en que se prestan los servicios públicos en un determinado país. Las diferencias en el número de empleados públicos entre países reflejan un complejo conjunto de factores, relacionados con las preferencias sociales y culturales de cada uno de ellos en el alcance, nivel y método de provisión de los servicios y bienes públicos (OCDE, 2009; Montesinos, Pérez y Ramos, 2014). Por tanto, un menor peso del empleo de las Administraciones Públicas en el total de la economía en un determinado país y en un momento del tiempo, no necesariamente conlleva un menor nivel de gasto público. Desde ese punto de vista, el peso de las Administraciones Públicas como empleadoras tampoco es un indicador exhaustivo del grado de incidencia de la actividad del sector público en la economía, dado que esta viene determinada por los factores anteriormente mencionados, a los que habría que añadir otros de carácter institucional y de política económica, relacionados, por ejemplo, con las políticas de subvenciones, transferencias sociales o grado de colaboración público\#privada (Martínez Matute y Pérez, 2017).

Para conocer si España tiene muchos o pocos empleados públicos hay que mirar a los países de su entorno. El porcentaje de empleados públicos es del 15\% en España similar al de Portugal (país con una población mucho más pequeña) e inferior al 29\% en Suecia o al 22\% de Francia. Otros países como Italia (14\%) o Alemania (10\%) cuentan con menos empleados públicos. La media comunitaria es del 16\%. España, por tanto, está próxima a la media europea.

El gasto salarial de las Administraciones Públicas (AAPP) en España supuso en 2016, según la Contabilidad Nacional (CN), algo más del $20 \%$ de la remuneración de los asalariados del total de la economía, y un $25 \%$ del gasto público total, mientras que el porcentaje de asalariados públicos sobre el total de empleados de la economía se situó por encima del $15 \%$. Estas cifras resultan similares a las de las principales economías avanzadas de la OCDE (Martínez Matute y Pérez, 2017). Las AAPP son, por tanto, actores relevantes del mercado de trabajo nacional. Sus decisiones en materia de personal influyen en la dirección de los flujos de trabajadores entre sectores económicos, en los procesos de fijación de los salarios del conjunto de la economía y, en definitiva, en la evolución económica agregada (Hernández de Cos, Moral Benito y Pérez (2016) y Hernández de Cos y Pérez, (2015).

Desde una perspectiva temporal amplía el número de empleados de las AAPP aumentó de forma sostenida desde 1987, a un ritmo superior al del incremento de la población, en particular hasta principios de la década de 2000. Este proceso se encuentra asociado, en gran parte, a la expansión del estado del bienestar, los servicios 
públicos y las transferencias competenciales a las CCAA. En los primeros años de la crisis económica reciente, entre 2009 y 2011 se produjo un aumento del número de empleados públicos, a diferencia de lo ocurrido en la mayoría de los países de nuestro entorno (Pérez et al., 2016). La intensificación de las restricciones a partir de 2010 llevó a que el empleo público se redujera en 2012 y 2013, y se estabilizara en 2014.

Con la crisis muy presente, las medidas de austeridad se convirtieron en una cuestión de gran importancia en la agenda política desde 2011. Estas medidas de austeridad han incluido la completa congelación de la contratación entre 2012 y 2014 ( $0 \%$ de tasa de reposición, excepto para los servicios esenciales a los que se aplica el 10\%). El 66\% de esas reducciones han sido en contratos temporales y la mayor parte de las mismas se han dado a escala de las Comunidades Autónomas (CCAA) (Gobierno de España, 2013).

Según la Encuesta de Población Activa (EPA), el empleo público presentó su nivel histórico absoluto más elevado en 2011, como se puede ver en el gráfico 1, alcanzando los 3,1 millones, tras registrar un incremento de 310.000 personas entre 2007 y 2011. En 2012 y 2013 se produjo una reducción de cerca de 350.000 efectivos, parte de los cuales se recuperaron entre 2014 y 2016. En este último año el número de empleados al servicio de las AAPP se situó algo por encima de los 2.840.000 trabajadores, un nivel ligeramente superior al observado en 2007 (en 30.000 puestos) (Martínez Matute y Pérez, 2017).

3.000 .000

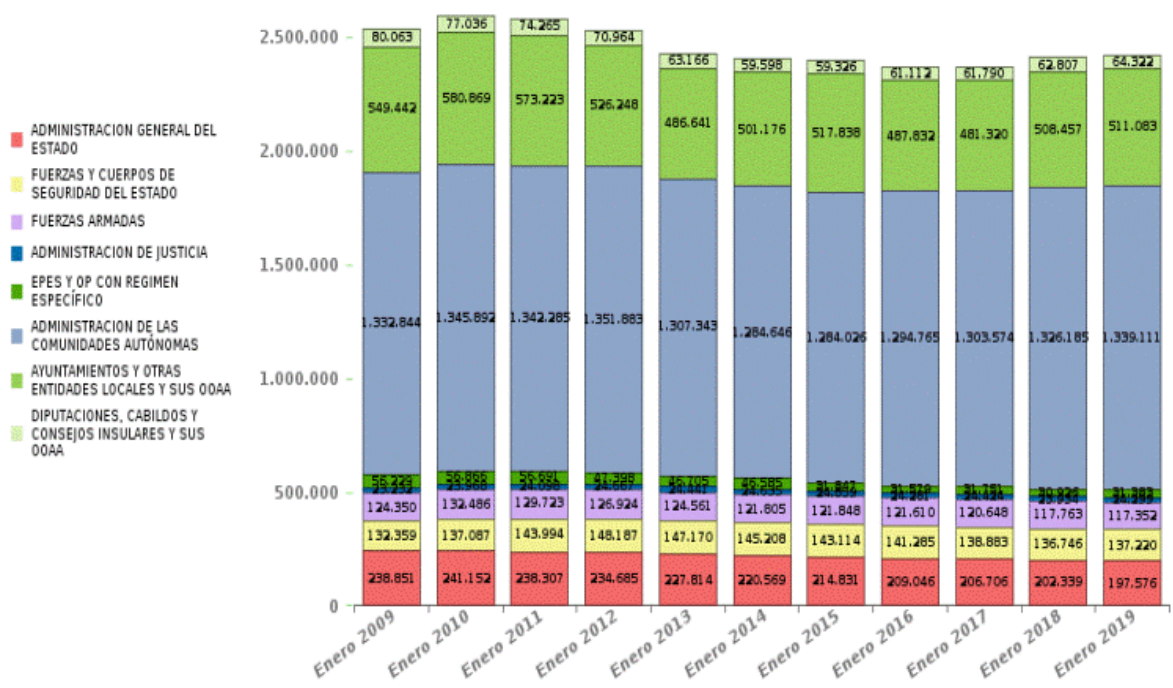

Gráfico 1.- Evolución del personal al servicio de las administraciones públicas Fuente: Registro Central de Personal (2019).

Por tipo de Administración (ver gráfico 2), en el año 2019, el 51,92\% del empleo de las AAPP españolas correspondió a las Comunidades Autónomas (CCAA), con 1.339.111 empleados; el 22,32 \%, a las Corporaciones Locales (CCLL), con 575.405, y el 7,66\% restante, a la Administración General del Estado, incluyendo a la Seguridad Social (AC), con 202.339 

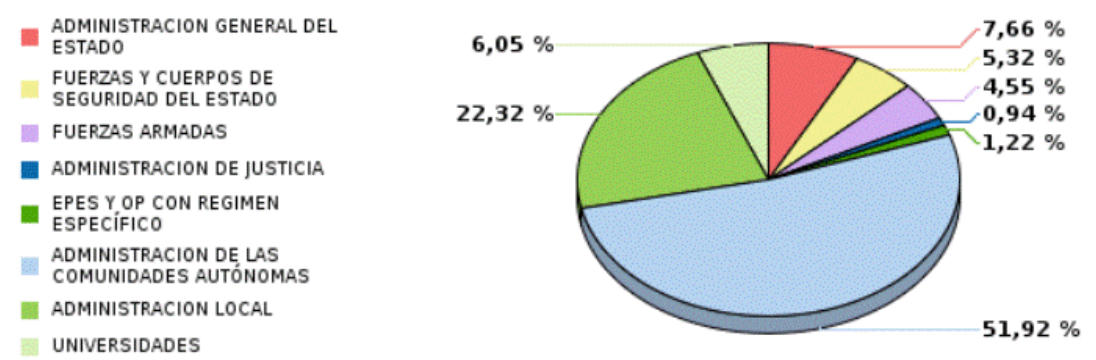

Gráfico 2.- Personal al Servicio de las Administraciones Públicas Fuente: Registro Central de Personal (2019).

En cuanto a la distribución territorial de efectivos por Comunidades Autónomas, Andalucía que es la comunidad de mayor tamaño es la que tiene un mayor número (460.702), seguida de la de Madrid (391.799) y Cataluña (316.759).

\section{MAPA CON LA DISTRIBUCIÓN TERRITORIAL DE EFECTIVOS POR ADMINISTRACIÓN PÚBLICA}

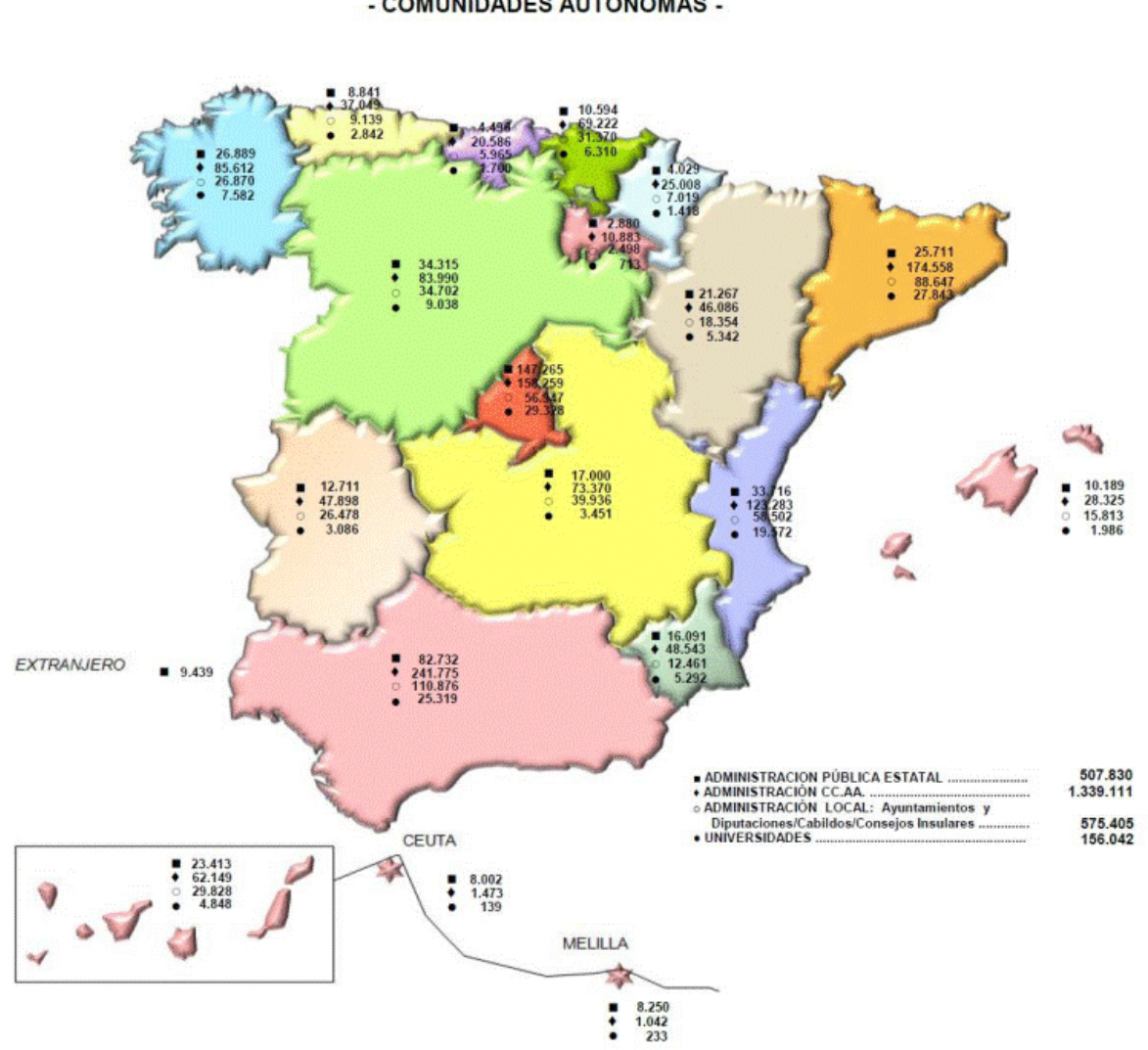

Fuente: Registro Central de Personal (2019).

Por tipo de personal en el grafico 3 se puede ver que más de la mitad es personal funcionario. Si vemos el porcentaje de personal funcionario por nivel de gobierno, en el central es del $81,55 \%$, en las CCAA del $58,45 \%$ y en los municipios del $34,43 \%$. 


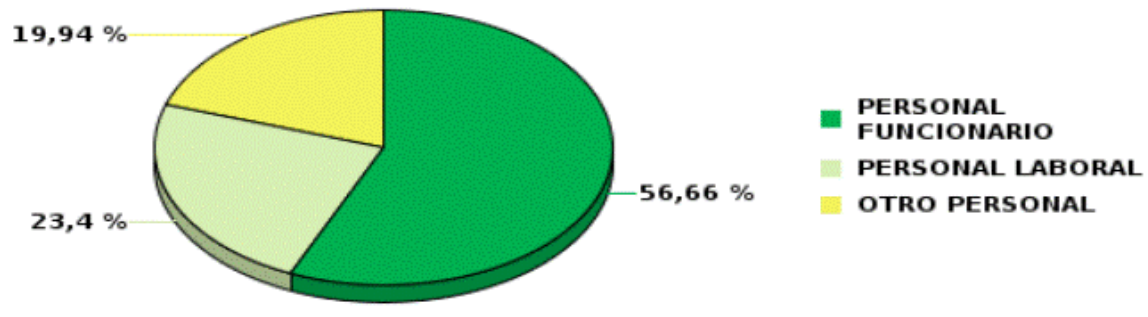

\section{Gráfico 3. Personal al servicio de las administraciones públicas por tipología de personal Fuente: Registro Central de Personal (2019).}

Un informe de Rastand España de 2018 señala que el 24,92\% de los trabajadores públicos son temporales, lo que supone el porcentaje más elevado desde el último trimestre de 2009. En la actualidad, hay 3.117.800 trabajadores que desarrollan su actividad en el sector público, de los que 777.100 lo hacen mediante un contrato temporal. Cuando se analiza la última década, se detecta que entre 2008 y 2009 se registraron los valores más elevados, al situarse el porcentaje de trabajadores del sector público con contrato de duración determinada entre el $25 \%$ y el $27 \%$. Desde entonces, este porcentaje descendió paulatinamente hasta alcanzar su mínimo en el primer trimestre de 2013, cuando se situó en el 19\%, con una cifra por debajo de los 560.000 ocupados. Desde entonces, a causa de las sucesivas restricciones de las ofertas de empleo público en el conjunto de las Administraciones, impuestas por los límites presupuestarios a la tasa de reposición, ha aumentado tanto el volumen total del empleo temporal (actualmente 777.100 trabajadores), como su peso sobre el total, que roza el $25 \%$.

La alta tasa de temporalidad en la administración pública española es producto de una variada combinación de factores que van desde fenómenos de clientelismo latente, pasando por algunas prácticas inadecuadas de gestión intergubernamental, como la perversa dinámica de subvenciones condicionadas dirigidas a financiar servicios, típicas entre los niveles autonómico y local. Pero también habría que incluir otros rasgos, como la inveterada desconfianza en nuestro sistema de selección de funcionarios, formal, garantista y escorado hacia lo memorístico que genera, como práctica habitual, la selección de personal no permanente al que en la práctica, primero se "prueba" y luego "se le saca la plaza", en una práctica convertida en una suerte de "rito de paso" o finalmente, las bajas capacidades de gestión de las organizaciones públicas en un contexto juridificado en las que el personal no personal no permanente juega el papel flexibilizador que no se obtiene con el fijo ${ }^{[\mathrm{v}]}$.

También han influido en el crecimiento de la temporalidad y de la precarización en la provisión de servicios del Estado del bienestar la aplicación de la tasa de reposición y la congelación de las ofertas de empleo público; recurso creciente a encargos a medios propios con pérdida del control de procesos y del conocimiento interno de la organización; envejecimiento de plantillas y limitación de la capacidad de innovación, todo ello en un entorno crecientemente inestable, competitivo y tecnificado en el que las organizaciones fían su capacidad de supervivencia a la captación de talento (Mapelli, 2018).

El Gobierno de Sánchez, para intentar revertir esta situación, lanzó a finales de marzo la mayor oferta de empleo público de la última década con 33.793 plazas de oposiciones, siendo esta la primera medida que se adopta para afrontar el envejecimiento de las plantillas. El grueso de esta oferta de empleo es para cubrir plazas vacantes de trabajadores que se han jubilado o han causado baja en los últimos años. 


\section{B) Selección}

La selección constituye una inversión a muy largo plazo, expresiva de las prioridades estratégicas de la organización, que debe ser optimizada de manera constante, en un contexto en el que las competencias requeridas para el desempeño de un puesto evolucionan rápidamente y se alejan de las definidas en el momento de realizar la selección. Por eso resulta esencial llevar a cabo una cuidadosa planificación a fin de determinar las funciones encomendadas a la organización, así como las profesiones y perfiles necesarios para atenderlas (Mapelli, 2018).

A lo largo de los próximos años las administraciones públicas van tener que competir con otras organizaciones en la captación de talento. Resulta obligado diseñar estrategias para conseguir atraer los mejores perfiles al sector público y hacerlo de modo que se garantice la legitimidad de la institución de la función pública mediante el refuerzo de su diversidad. Ha de incidirse igualmente sobre un modelo de selección que presenta importantes barreras de entrada debido al formato de algunas de las pruebas y que traslada íntegramente al aspirante el coste de la preparación. Por último, deben desarrollarse programas específicos para garantizar la representatividad de los colectivos con mayores dificultades de acceso a la función pública (Mapelli, 2018).

Los procesos selectivos de acceso a las Administraciones Públicas (AAPP) son una herramienta inevitable en la gestión de Recursos Humanos (RRHH) que si se usaran estratégicamente y como parte de un sistema integrado de organización y gestión de la diferencia podrían aportar muchos réditos para abordar realidades actuales de interinidad y de cercanas jubilaciones masivas de funcionarios en las AAPP. También por una nueva concepción de los cuerpos de funcionarios y por la trascendente distinción entre persona y puesto (Gorriti, 2018). Cuanto más se parezca el proceso selectivo al trabajo para el cual se hace la selección, mayor será la probabilidad de que dicho proceso selectivo sea válido y justo.

Toda buena selección implica la satisfacción de dos valores: la eficacia y la igualdad. Eficacia, ya que se trata de identificar aquellos trabajadores/as que serán los más idóneos para desempeñar las tareas del puesto para el que se selecciona. Igualdad, para que personas con las mismas posibilidades, tengan las mismas probabilidades. Para que todos aquellos que se presenten al proceso selectivo sean medidos con los mismos instrumentos, en el mismo orden, con los mismos contenidos, y con las mismas posibilidades de éxito (Gorriti, 2018).

Algunos elementos constitutivos de los perfiles de los puestos son válidos para todos los puestos y para

todas las culturas: la habilidad mental general (inteligencia), el factor conciencia de la personalidad ${ }^{\text {[vi] }}$ y la integridad. Este último es un compuesto de distintos factores de personalidad (Schmidt \& Hunter, 1998; Salgado, Viswesvaran y Ones, 2001; Ones, Viswesvaran y Dichert, 2005).

Los retos que actualmente tiene el empleo público en España ponen en evidencia lo que Jiménez Asensio (2017) ha descrito como una situación de "fragilidad del sistema de mérito", especialmente preocupante en el ámbito de la selección, tal y como analizan estudios recientes (Crespo y Polo, 2015; Areses Vidal et al., 2017; Losada et al., 2017). Ello lleva a reflexionar sobre la necesidad de repensar la vigente configuración institucional del sistema selectivo que, como recuerda Ramió (2018), tras la larga crisis ofrece signos claros de agotamiento para poder hacer frente las transformaciones que el sector público debe acometer en los próximos años.

Otro problema muy grave es, en concreto, el acceso al empleo público mediante los denominados "procesos de consolidación” o como los califica Jiménez Asensio “aplantillamiento" de un enorme grupo de empleados públicos que han accedido al empleo público mediante unos sistemas de acceso sin las más mínimas garantías y que además, en muchos casos con una clara vinculación política y sindical y dudosa cualificación profesional, lo que ha originado una pérdida de talento y profesionalidad en las administraciones, y a su vez un fraude para el resto de los ciudadanos que han visto defraudadas sus expectativas de acceso al empleo público

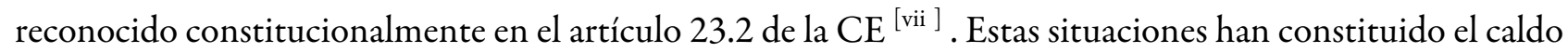
de cultivo perfecto para el florecimiento y desarrollo de la corrupción, fenómeno que afecta a todas las 
administraciones públicas y a todos sus niveles, de manera que solo desvinculando la politización del empleo público e incorporando la exigencia de unos principios de ética pública a todos los empleados públicos se podrá comenzar un nuevo camino ya despejado y acorde con el orden axiológico constitucional. (Fondevila, 2018).

Lo que tampoco parece razonable es que el modelo de las pruebas de acceso a la función pública no se hayan modificado prácticamente en los últimos 40 años, salvo en algunos aspectos puntuales y poco relevantes como la sustitución de unos temarios por otros. Las oposiciones a altos cuerpos no son justas desde el punto de vista social, pues favorecen a aquellos candidatos de familias que pueden permitirse el coste de oportunidad de pasar varios años estudiando sin necesidad de trabajar, además de los costes derivados de la propia preparación ya sea vía academia o vía preparador particular. Esto tiene la consecuencia de seleccionar a personas que no son representativas de todos los grupos sociales lo que puede contribuir a deslegitimar la función pública en sus escalones superiores por su falta de diversidad (Fernández-Villaverde e Ibañez, 2017). Además, tampoco se genera la adecuada "diversidad intelectual" en la medida en que tienden a seleccionar a gente con un perfil muy similar. Habiendo, como señalan varios autores, otros sistemas de acceso igualmente meritocráticos que minimizan estos costes.

Las exigencias constitucionales de igualdad, mérito y capacidad que garantizan los sistemas selectivos generan ciertas rigidices, como la lentitud de adaptar los procesos a las competencias o necesidades emergentes, y notables desfases, como el excesivo peso de las pruebas de conocimientos frente a las que permiten evaluar las competencias de los aspirantes. Habiendo recibido muchas críticas el sistema por la falta de medición de la fiabilidad y validez de las pruebas (Salgado y Moscoso, 2008; Gorriti, 2011; Jiménez Asensio, 2018), así como por sus elevados costes (Mapelli, 2018).

Otro problema es la inexistencia de datos de calidad, al menos de procedencia oficial, que evalúen el mayor o menor éxito de esos procesos de reclutamiento desde distintos puntos de vista: procedencia de los opositores, diversidad, número de años de preparación, tiempo de permanencia en el sector público después de superada la selección, carrera profesional, formación posterior en Escuelas de Administración, formación continua, excedencias, etc. De nuevo, la Administración carece de datos fiables sobre el éxito mayor o menor - medido desde distintas perspectivas - de sus propios procesos de reclutamiento. La recopilación y el análisis de esos datos es esencial para que la Administración Pública disponga de información de calidad sobre la captación y mantenimiento del talento que tanto necesita (Nuez, 2018).

Algunas de las medidas necesarias, según Fondevila (2018) para la incorporación de cambios en la selección, son: a) la creación de órganos especializados en técnicas de selección, con un carácter absolutamente técnico, profesionalizado e independiente[1] ${ }^{[\text {viii] }}$, que permitan una correcta implementación de unos nuevos tipos de pruebas; b) estos órganos de selección deben tener carácter permanente y no una duración limitada temporalmente ad hoc; c) deben elaborarse protocolos formalizados de las técnicas de selección y criterios de evaluación a utilizar, con los correspondientes perfiles. Estos instrumentos deberían ser de uso común por todos los órganos técnicos de selección; d) la centralización de los procesos de selección en las escuelas de formación de funcionarios lo que permite abaratar los costes económicos, el mayor inconveniente para realizar los cambios.

\section{C) Formación}

El conocimiento y la formación continua de los trabajadores es un elemento estratégico para todos los profesionales y organizaciones, si consideramos que su desarrollo permite proporcionar mejores respuestas delante de un entorno complejo y cambiante. No obstante, la información que hoy tenemos sobre la manera como aprendemos individual y colectivamente exige revisar los tradicionales sistemas y métodos de formación. Frente a formatos presenciales y basados en la imitación, se proponen, cada vez más y para 
la formación continua, alternativas que exigen mayores esfuerzos colaborativos, que permitan incorporar y compartir el conocimiento personal, el conocimiento institucional y el conocimiento social, promoviendo nuevo conocimiento y una gestión adecuada del mismo (Martínez, 2016).

El modelo organizativo de formación en España es de dispersión. El resultado de este modelo institucional de formación es evidente: por un lado, se observa una clara fragmentación de la formación en diferentes niveles e instancias, así como una reiteración de programas que duplican gasto y pueden ser calificados, en buena medida, de ineficientes, aportando datos más que dudosos sobre su transferencia efectiva al empleo público; por otro, salvo las Escuelas e Institutos de Administración Pública, no hay entidades que acrediten la calidad de los cursos impartidos y, sobre todo, la adecuación de los contenidos a las necesidades de la organización. Jiménez Asensio (2010) ya señalaba que el modelo existente de formación de empleados públicos está atomizado, es dudosamente eficiente y representa unos costes financieros desmesurados para la escasa rentabilidad que la organización recibe de esa formación vía transferencia de conocimientos.

En la Administración General del Estado existe el Instituto Nacional de Administración Pública (INAP) como centro de formación de los cuerpos generales y locales de habilitación nacional. Además, existen diversas escuelas o institutos por cada ámbito funcionarial. A nivel de las Comunidades Autónomas (CCAA) varían entre un centro único para todo tipo de personal y centros más o menos especializados. En el ámbito local la casuística en mucho mayor, además de los planes que ofrece el INAP por vía del antiguo Instituto de Estudios de Administración Local, cabe incluir las actividades formativas que organizan los propios ayuntamientos y diputaciones, además de las CCAA. También imparten formación los agentes sociales (sindicatos) y las asociaciones o federaciones de municipios y provincias, además de la formación que puedan impartir fundaciones, entidades privadas (como, por ejemplo, Escuelas de Negocios) o las propias Universidades a través de los títulos de postgrado y de la innumerable oferta de títulos propios.

La formación cada vez más comienza a ser entendida no como premio o como "asunto escolar individual" por decirlo con las palabras de Crozier (1989), sino como prioridad de la política de empleo público, como palanca de cambio en la que la transferencia del aprendizaje al puesto de trabajo debe ser el objetivo principal, lo que conlleva revisar la propia oferta existente, abandonando el "formato catálogo" (Jiménez Asensio, 2010), innovando en los sistemas de determinación de necesidades y dando cabida al reconocimiento del aprendizaje no formal como medio de desarrollo. La formación entendida como una estrategia capaz de dotar a las personas de la organización de las competencias necesarias para encontrar y aplicar soluciones adecuadas a las nuevas demandas y posibilidades tanto de la propia organización como del servicio que presta a la ciudadanía.

Existiendo la necesidad de ofrecer formación que favorezca - más allá de los incentivos que también se pueden establecer en el mismo sentido - determinados comportamientos de los empleados públicos, tales como la asunción de riesgos y responsabilidades, la gestión de conflictos de intereses contrapuestos o la gestión de equipos diversos y pluridisciplinares (Nuez, 2018), también debe de tener un elevado componente práctico e internacional. Es indudable que hay que evaluar también el resultado de esta formación con mucho más rigor que hasta ahora tanto desde el punto de vista de su utilidad para el personal empleado público como, sobre todo, de su utilidad para la organización (Nuez, 2018).

\section{CONCLUSIONES}

La complejidad cada mayor de las políticas y servicios públicos unidos a los cambios sociales y tecnológicos hacen cada vez más necesarios cambios en la gestión de los recursos humanos públicos. Elementos, por otro lado, fundamentales en una gestión de calidad. En todos los países, a pesar de sus diferencias de tamaño o de desarrollo, se plantean similares problemas y retos de futuro para una gestión de recursos humanos públicos que pueda dar respuesta a los cambios que se plantean. 
Por ahora, en España y Brasil, sigue sin implantarse de modo efectivo un enfoque sostenido de gestión a medio plazo, circunstancia a la que no es ajena la dificultad de arraigo de la propia gestión pública de recursos humanos (GPRH) como disciplina aplicada a un entorno institucional, el público, caracterizado por unos criterios de éxito distintos al del mercado, sociales y políticos además de económicos, y con unos actores singulares - políticos y sindicales - y un marco jurídico que trata de asegurar valores específicos como el mérito. La influencia que el pasado patrimonialista ejerció sobre la estructura de las administraciones de los dos países ha impedido la consagración de un elemento clave para la correcta gestión del sector público, que es la percepción de la diferencia entre lo público y lo privado, persistiendo en ellas el clientelismo, y los corporativismos. Además, en el caso de Brasil, existe la ausencia de una burocracia profesionalizada en dimensiones adecuadas para el tamaño del país y con un reparto igualitario por todo el territorio.

En lo que respecta al número de empleados públicos la media de España, que es del 15\%, está próxima a la media europea, que es del 16\%. Aunque en los años de crisis económica se redujo el número de contrataciones y de reposición de efectivos, la situación comienza a cambiar con la mayor oferta de empleo público de la última década presentada por el Gobierno Sánchez, con 33.793 plazas de oposiciones. En Brasil tampoco existe un número elevado de funcionarios públicos si lo comparamos con países de América Latina o Europa y con su población, lo que si se produce es una desigualdad en la distribución de los empleados en cada área. Existiendo desproporción en el número de funcionarios entre las áreas urbanas y rurales, entre las capitales y el resto del país y entre los servicios del centro y de la periferia.

Los procesos selectivos de acceso a las Administraciones Públicas (AAPP) son una herramienta inevitable en la gestión de Recursos Humanos (RRHH) que si se usaran estratégicamente y como parte de un sistema integrado de organización y gestión de la diferencia podrían aportar muchos réditos para abordar realidades actuales y de futuro. Para ello se hace necesario en España repensar el sistema de selección a partir de la creación de un organismo especializado en selección, la adaptación del formato de las pruebas de modo que permitan medir adecuadamente las competencias de los aspirantes, y el aprovechamiento de todo el potencial de las escuelas de formación de las administraciones. También las administraciones deben disponer de herramientas para evaluar el rendimiento, identificar las áreas preferentes de formación y ofrecer asesoramiento individualizado para el desarrollo de itinerarios profesionales que permitan a los empleados públicos alcanzar su pleno potencial.

Aun contando con una percepción ciudadana positiva del sistema de concursos, Brasil debe realizar cambios para subsanar las debilidades percibidas por el Ministerio de Planificación, Presupuesto y Gestión en el proceso de ingreso (o en las etapas posteriores a éste): a) elevada judicialización de los concursos, en el 52\% de los casos con referencia a problemas relativo al proceso de elaboración y publicación de la convocatoria ("edital"); b) demora en el tiempo entre la autorización emitida por el ministerio para la realización del concurso y el ingreso en el servicio civil; c) elevada rotación de los seleccionados - que participan en varios concursos a la vez - y rotan, en el corto plazo, de puestos de más baja remuneración a otros de remuneración más alta, sin demostrar interés en una carrera específica y; d) efectividad debatible, en la medida que no necesariamente se selecciona al candidato de perfil más adecuado al puesto en cuestión sino el que obtiene una mejor calificación en los exámenes.

En la formación, otro de los elementos importantes de la gestión de recursos humanos, es esencial, en los dos países, la combinación de acciones formativas con la generación de actividades que facilite, tanto su configuración concreta como su desarrollo efectivo adaptado a la realidad organizativa. Los nuevos escenarios formativos que se planteen deben estar asociados especialmente a la formación on line, a la formación colaborativa, al autoaprendizaje y al aprendizaje informal, además de relacionarse con una formación más personalizada y adecuada a las necesidades de los empleados públicos. Sin olvidarnos de reorganizar los centros de formación para que sean más eficientes y de evaluar el resultado de esta formación con mucho más rigor que hasta ahora, tanto desde el punto de vista de su utilidad para el personal empleado público como, sobre todo, de su utilidad para la organización 
Por último, hay que ser también muy conscientes de que será necesario un amplio periodo de transición en que convivan lo viejo y lo nuevo. En este sentido, todo periodo de transitoriedad requiere de una gestión específica y cuidadosa que debe de planificarse adecuadamente para que el cambio sea incremental y asumible y no genere resistencias. Hay que entender que durante este periodo deben de convivir dos tipos de funcionarios de perfiles muy distintos y que los dos van a ser necesarios para hacer posible la trasmisión del know how imprescindible. También la cultura organizativa debe de ir cambiando de manera paulatina pero sin que nadie se sienta atacado o relegado. Sólo de esta forma será posible la transición de la Administración que hoy conocemos a la nueva Administración que necesitamos.

\section{REFERENCIAS}

Arroyo Yanes, L.M. (2016). Los instrumentos de gestión del empleo público. Madrid. INAP.

Blondel, J. (2005). The central role of comparative politics in political analysis. Scandinavian Political Studies, 28(2), $183-191$.

Bulcourf, P. A., y Cardozo, N. D. (2008). ¿Por qué comparar políticas públicas?. Argentina: Documento de Trabajo \# 3.

Cofer, David A. (2000). Informal workplace learning (Practical Application Brief No. 10). Columbus, OH: Center on Education and Training for Employment.

Comisión para la Reforma de las Administraciones (CORA). (2013). Informe Cora.

Crozier, M. (1989). Estado modesto, Estado moderno. Estrategia para el cambio. México: FCE.

Cuenca, J.J. (2018). Instrumentos de planificación de recursos humanos y selección ¡cambio de paradigma? Revista Vasca de Gestión de Personas y Organizaciones Públicas No. especial 2: 52-65.

Davenport, T. H. y Prusak, L. (1998). Working knowledge: How organizations manage what they know. Boston, EUA: Harvard Business School Press.

Doeringer, P.B. y Piore, M.J. (1983) Los mercados internos de trabajo, en L. Toharia (comp.) El mercado de trabajo: teoría y aplicaciones, Madrid, Alianza Universidad.

Estatuto Básico del Empleado Público (EBEP) (2007). Ley 7/2007, de 12 de abril.

Fernández-Villaverde, J. e Ibáñez Colomo, P. (2017). Sobre las oposiciones a altos cuerpos. [Entrada blog] Disponible en: http:// nadaesgratis.es/ fernandez-villaverde/sobre-lasoposiciones-a-altos-cuerpos [Fecha de consulta: 07/11/18].

Fondevila, J. (2008). Selección y Pérdida de la condición de empleado público. Barcelona: ATELIER.

Fondevilla, J. (2018). Repensar el diseño de los procesos selectivos en el empleo público: respuestas ágiles frente a las necesidades inmediatas y fortalecimiento de la especialización de los órganos de selección. Revista Vasca de Gestión de Personas y Organizaciones Públicas No. especial 2:98-111.

Gobierno de España, Ministerio de Empleo y Seguridad Social (2013). Report evaluating the impact of the labour reform, Gobierno de España, Madrid, www.empleo. gob.es/es/destacados/HOME/impacto_reforma_laboral/ Report_evaluating_the_impact_of_the_Labour_Reform.pdf.

Gorriti, M. (2011). Sistema de selección para el acceso a una función pública postburocrática. Anuario multidisciplinar para la modernización de las Administraciones Públicas, 7, 147-161.

Gorriti, M. (2016). Análisis cognitivo de tareas. Gestión de conocimientos en la Administración General del País Vasco. Disponible en: https://www.google. es/search?q=gorriti+an\%C3\%A1lisis+cognitivo + tareas\&rlz=1C1AVNG_enES719ES719\&oq=gorriti+an\%C3\%A1lisis+cognitivo +tareas\&aqs=chrome..69i57.11221j1j7\&sourceid=chrome\&ie=UTF-8\# [Fecha de consulta: $07 / 11 / 18$ ]

Gorriti, M. (2018). Innovar en selección desde la evidencia empírica y las nuevas competencias. Revista Vasca de Gestión de Personas y Organizaciones Públicas No. especial 2: 65-85.

Gorriti, M. y Jiménez Asensio, R. (2016). Marchitar o florecer. La función pública ante el reto del envejecimiento de las plantillas. Disponible en: http://HYPERLINK http://www.lamiradainstitucional.com [Fecha de consulta: $17 / 10 / 18]$ 
Gorriti, M. y Jiménez Asensio, R. (2018). Mantener o transformar (gestión inteligente de vacantes en el empleo público del futuro). Disponible en: https://rafaeljimenezasensio.com/2018/09/25/mantener-o-transformargestion-inteligente-de-vacantes-en-el-empleo-publico-del-futuro/ [Fecha de consulta: 10/08/18]

Grandinetti, R.M. (2003). La incorporación de tecnologías a la gestión local:¿ capacidad para la gestión relacional?. Revista de Ciencias Sociales 9 (2): 191-204.

Grau, M., y Mateos, A. (2002). Análisis de políticas públicas en España: enfoques y casos. Valencia: Tirant lo Blanch.

Hernández de Cos, P.; Moral Benito, E. y Pérez, J.J. (2016). El empleo y los salarios públicos durante la crisis: análisis desde una perspectiva internacional y regional, Papeles de Economía Española, 147: 68\#91.

Hernádez de Cos, P. y Pérez, J.J. (2015). El impacto de los salarios y el empleo público. Una perspectiva macroeconómica, Presupuesto y Gasto Público, 79:11\#26.

Hernandez Sampieri, R., Fernandez Collado, C., Baptista Lucio, P. (2006). Metodología de la Investigación. 4a Edicion. Mexico: McGraw-Hill, Interamericana Editores.

Iacoviello, M. (2006). Análisis comparativo por subsistemas. En K. Echebarría (ed.), Informe sobre la situación del Servicio Civil en Latinoamérica, Washington, DC, Estados Unidos: BID.

Jiménez Asensio, R. (2010). La formación de los empleados públicos tras el EBEP: ¿cambio de paradigma?. Revista Vasca de Administración Pública, 87-88: 637-688.

- - - (2017). La fragilidad del sistema de mérito I, II, III y IV. [Entrada blog]. Disponible en: https:// estudiosectorpublico.com/blog/ [Fecha de consulta: 10/09/18].

- - - (2018). Función pública, ¿transformación o crisis? (i): la herencia recibida. [Entrada blog]. Disponible en: https://rafaeljimenezasensio.com/inicio-3/ [Fecha de consulta: 10/09/18].

Kamieniecki, S., y Sanasarian, E. (1990). Conducting comparative research on environmental policy. Natural Resources Journal, 30(2), 321-339.

Lohman, M. C. (2005). A survey of factors influencing the engagement of two professional groups in informal workplace learning activities. Human Resource Development Quarterly, 16(4), 501-527.

Lohman, M. C. (2009). A Survey of Factors Influencing the Engagement of Information Technology Professionals in Informal Learning Activities. Information Technology, Learning, and Performance Journal, 25 (1), 43-53

Longo, F. (2004). Mérito y flexibilidad. La gestión de las personas en las organizaciones del sector público. Barcelona: Paidós.

Mapelli, C. (2018). La visión comparada: nuevos sistemas de selección de la alta función pública en las democracias avanzadas. Revista Vasca de Gestión de Personas y Organizaciones Públicas No. especial 2: 32-50.

Martínez, J. (2016). Nuevos modelos de formación para empleados públicos. Guía para la transformación. Barcelona: UOC.

Martínez Matute, M. y Pérez, J.J. (2017). La evolución del empleo de las administraciones públicas en la última década. Banco de España.

Mauri, J. (2009). Análisis general del Estatuto básico del empleado público: problemas, soluciones y huidas. QDL.

Ministerio de Política Territorial y Función Pública (2018). Boletín Estadístico del Personal al Servicio de las Administraciones Públicas. Registro Central de Personal.

Montesinos, A.; Pérez, J.J. y Ramos, R. (2014). El empleo de las Administraciones Públicas en España: caracterización y evolución durante la crisis, Documentos Ocasionales, n. ${ }^{\circ}$ 1402, Banco de España.

Morin, E. (2011). Introducción al pensamiento complejo. Gedisa Editorial.

Munck, G. L. (2006). The past and present of comparative politics. California: Kellogg Institute for International Studies.

Nuez Sánchez-Casado, E. (2018). Acceso a la función pública: atraer talento y cambiar el modelo. Revista Vasca de Gestión de Personas y Organizaciones Públicas No. especial 2: 86-97.

OCDE (2009). Employment in general government and public corporations en Government at a Glance 2009, OECD Publishing. 
_-_. (2016), Panorama de las Administraciones Públicas: América Latina y el Caribe 2017, Éditions OCDE, Paris. - - . (2017). Skills for a High Performing Civil Service, Estudios de Gobernanza Pública. Paris: Servicio de Publicaciones de la OCDE.

Ones, D. S., Viswesvaran, C. y Dilchert, S., (2005). Personality at work: raising awareness and correcting misconceptions. Human Performance, 18 (4): 389-404.

Parsons, W. (2008). Políticas Públicas. México: Miño Dávila.

Pérez, J. J.; Aouriri, M; Campos, M.; Celov, D.; Depalo, D.; Papapetrou, E.; Pesliakaite, J.; Ramos, R. y Rodríguez, M. (2016). The fiscal and macroeconomic effects of government wages and employment reform, European Central Bank Occasional Paper Series, n. ${ }^{\circ}$ 176, agosto.

Ramió, C. (2018). Los nuevos principios de un modelo de gestión del empleo público en España para una administración pública sostenible y contingente. Working Papers Nueva Serie 79-82: 1-32.

Ramió, C. y Salvador, M. (2018). La nueva gestión del empleo público. Recursos Humanos en Innovación de la Administración Barcelona: Tibidabo Ediciones.

Rastand (2018). Informe sobre empleo en España.

Salgado, J.F., Moscoso, S. (2008). Selección de personal en la empresa y las administraciones públicas: de la visión tradicional a la visión estratégica. Papeles del psicólogo, vol. 29, 1, 16-24.

Salgado, J.F., Viswesvaran, C. y Ones, D.S. (2001). Predictors used for personnel selection: an overview of construct, methods and technichs. En N. Anderson, D.S. Ones, H.K. Sinangil y C. Viswesvaran (Eds.), Handbook of Industrial, Work and Organizacional Psychology. Vol 1:165-199. Londres: Sage.

Schmidt, F.L. y Hunter, J.E. (1998). The validity and utility of selection methods in personnel psychology: Practical and theoretical implications of 85 years of research findings. Psychological Bulletin, 124: 262-274.

Selltiz, C., Wrightsman, L.S. y Cook, S.W. (1980). Métodos de Investigación en las Relaciones Sociales. Madrid: Rialp. Tamames, R. (2018). ¿Qué Robot se ha Llevado mi Queso? Barcelona: Alienta Editorial.

Villoria, M. y. Del Pino, E. (2009). Dirección y Gestión de Recursos Humanos en las Administraciones Públicas, Tecnos, Madrid, 3. ${ }^{\text {e edición. }}$

\section{Notas}

ILa noción normativa de empleado público es relativamente reciente (2007), mientras que sobre la noción de funcionario público es a través de la cual se ha construido la Administración Pública Española.

II El Gobierno de Rodríguez Zapatero limitó la tasa de reposición —las sustituciones por cada baja - al 30\% en 2009. Es decir, que si un año se jubilaban 100 funcionarios solo eran reemplazados por 30 nuevos empleados. Rajoy también restringió la tasa de reposición durante sus seis años de mandato. De hecho, hasta 2016 eliminó las reposiciones. No se sustituían los empleados que se jubilaban salvo los de sectores esenciales.

El resultado de prolongar este mecanismo de ajuste durante 10 años ha derivado en una plantilla envejecida, con más carga de trabajo y abocada a una gran renovación o restructuración.

IIILas administraciones públicas en España van a jubilar durante los próximos 15 años a un millón de empleados públicos (Ramió y Salvador, 2018: 246), entre los cuales hay más de un 80 por ciento de su personal directivo y técnico superior.

IVVéase M. Villoria y E. Del Pino, Dirección y Gestión de Recursos Humanos en las Administraciones Públicas, Tecnos, Madrid, 3. ${ }^{a}$ edición, 2009, pp. 434 y ss.

V Es la conocida hipótesis del «mercado laboral secundario » típica de entornos laborales rígidos enunciada por Doeringer y Piore (1983).

VI Hoy por hoy existe un consenso general respecto a que la personalidad está constituida por cinco grandes factores (conocidos como los «Big Five»), que a su vez, están constituidos por 30 rasgos. Los factores son los siguientes:

- Conciencia: alto deseo de logro, rigurosidad, orden y responsabilidad. Competencia.

— Estabilidad Emocional (Neuroticismo): control de emociones y resistencia a la presión. 
- Amigabilidad: gusto por las relaciones, generosidad y cooperación.

— Extroversión: asertividad (ser capaz de decir «no»), actividad y locuacidad, optimismo y energía.

- Apertura a la Experiencia: apertura de mente, tendencia a cuestiones conceptuales o intelectuales.

VII Para Fondevila $(2008 ; 2018)$ resulta incomprensible que se admita como un mérito «el tiempo de servicios prestados a la administración », dado que este supuesto mérito es una simple constatación de la duración de la relación de servicios, pero en ningún caso acredita la calidad de la prestación de los servicios.

VIII Las debilidades del modelo existente (Mauri, 2009; Arroyo Yanes, 2016; Fondevila, 2018) tienen que ver tanto con las probabilidades de acierto en la elección de los componentes de cada órgano de selección en cuanto a su capacitación como en la disponibilidad del tiempo necesario para dedicarse a una función tan exigente y relevante, teniendo en cuenta que normalmente lo deben compatibilizar parcialmente con sus responsabilidades como empleados públicos.

\section{BY-NC-ND}

